\title{
An Update on Arthritis in Canada - National and Provincial Data Regarding the Past, Present, and Future
}

\author{
Elizabeth M. Badley (D), Céline M. Goulart, Dov B. Millstone, and Anthony V. Perruccio (1)
}

\begin{abstract}
Objective. To provide updated arthritis estimates for Canada given a change in wording in the 2015 Canadian Community Health Survey (CCHS) arthritis question.

Methods. Prevalence data from the 2000 to 2016 CCHS were used to document trends in the prevalence of arthritis over time. Projections of arthritis prevalence were also calculated using data from CCHS 2015 in conjunction with Statistics Canada's published population projections. Data for 2015 were also used to provide summary data on the effect of arthritis.

Results. Between 2000 and 2014 there were some fluctuations in the prevalence of arthritis (age $\geq 15$ yrs), with the range of prevalence varying between $15.4 \%$ and $17.6 \%$. There was a significant increase in overall prevalence to over $20 \%$ with the 2015 and 2016 surveys (6 million Canadians), coinciding with a revised wording of the arthritis question. This increase was observed in all age and sex groups, except for men aged $85+$. The overall characteristics of the 2015 arthritis population were similar to those in 2007/08. Using the updated 2015 CCHS arthritis data, projection estimates suggest the population prevalence of arthritis will increase to just over $24 \%$ by 2040 , with the number of Canadians living with arthritis projected to increase by about 50\% from 2015 to 2040 .

Conclusion. The revised question likely increased ascertainment of arthritis owing to inclusion of examples of arthritis diagnoses in the CCHS question and more explicit wording in the French version, resulting in a large increase in the estimated prevalence and numbers of people with arthritis in Canada. (First Release February 15 2019; J Rheumatol 2019;46:579-86; doi:10.3899/jrheum.180147)
\end{abstract}

Key Indexing Terms: ARTHRITIS

\section{EPIDEMIOLOGY CANADIAN COMMUNITY HEALTH SURVEY (CCHS)}

PREVALENCE

Arthritis is a highly prevalent chronic disease in Canada with significant economic burden owing to the loss of productivity, disability, and increased healthcare use associated with the disease ${ }^{1,2}$. Up-to-date information on the prevalence and effect of arthritis in the population is important for policy making, raising public awareness, and informing funding allocations for arthritis care and research. The last readily available publication on the effect of arthritis in Canada was for 2007/08 in a report published by the Public Health Agency of Canada (PHAC) in $2010^{1}$.

The major source of national population-based data on

From the Dalla Lana School of Public Health, University of Toronto; Health Care and Outcomes Research, Krembil Research Institute,

University Health Network, Toronto, Ontario, Canada.

E.M. Badley, DPhil, Dalla Lana School of Public Health, University of Toronto, and Health Care and Outcomes Research, Krembil Research Institute, University Health Network; C.M. Goulart, MPH, Dalla Lana School of Public Health, University of Toronto; D.B. Millstone, MPH, Health Care and Outcomes Research, Krembil Research Institute,

University Health Network; A.V. Perruccio, PhD, Dalla Lana School of Public Health, University of Toronto, and Health Care and Outcomes Research and Arthritis Program, Krembil Research Institute, University Health Network, and Department of Surgery, Faculty of Medicine,

University of Toronto.

Address correspondence to Dr. E.M. Badley, Krembil Research Institute, Toronto Western Hospital, MP 10-310, 399 Bathurst St., Toronto, Ontario M5T2S8,Canada.E-mail: e.badley@utoronto.ca

Accepted for publication October 10, 2018. arthritis in Canada is Statistics Canada's Canadian Community Health Survey (CCHS $)^{3}$. This is an ongoing survey of a representative sample of the Canadian household population that started in 2000 . Arthritis is ascertained in the CCHS as one of the response options in a block of questions about "long-term health conditions which are expected to last or have already lasted 6 months or more and have been diagnosed by a health professional," to which respondents can answer yes or no. Statistics Canada periodically revises the CCHS methodology and questionnaire, including rephrasing the question concerning arthritis. There have been several changes (detailed below) since 2000, with a major change in wording of the arthritis question in 2015. Each change in wording has implications for estimates of prevalence and numbers of people with arthritis. The purpose of this paper is (1) to report how changes in the wording of the arthritis question have affected estimates of the prevalence of arthritis, (2) to provide summary data on the effect of arthritis to update the estimates provided in the 2010 PHAC report, and (3) to provide projections for the future prevalence and numbers of people with arthritis based on the 2015 estimate $^{1}$.

\section{MATERIALS AND METHODS}

Data source. The CCHS uses a cross-sectional multistage complex sampling design to obtain a nationally representative sample ${ }^{3}$. The target population for the surveys is individuals aged $\geq 12$ years living in private dwellings in

Personal non-commercial use only. The Journal of Rheumatology Copyright @ 2019 . All rights reserved. 
all 10 provinces and 3 territories of Canada. Institutional residents, full-time members of the Canadian Forces, those living in certain remote regions or reserves, and other Aboriginal settlements are excluded. The overall coverage of the CCHS is over $97 \%^{3}$. The CCHS initiative began in 2000. Details of the sampling and methodology of the surveys are available on the CCHS Web page ${ }^{4}$. Overall response rates for the CCHS from 2000 to 2016 ranged from $57.5 \%$ to $78.9 \%$.

Data for the population aged $\geq 15$ years were used for the present analyses. Arthritis prevalence data from the 2000-2016 surveys were used to document trends in the prevalence over time.

Data for 2015 were used for projections of arthritis prevalence in conjunction with Statistics Canada's published population projections for Canada. For Statistics Canada's projections, births, deaths, and migration factors are used as variables for population growth predictions, with 3 scenarios: high, medium, and low growth. We used the medium population growth scenarios, which have constant medium fertility, medium life expectancies, and medium annual immigration levels.

Data for 2015 were also used to inform summary data on the effect of arthritis, because the 2015 CCHS has a more comprehensive set of arthritis-relevant variables than the more recent 2016 survey.

Arthritis questions. Prior to 2007, the arthritis question in English was, "Do you have arthritis or rheumatism, excluding fibromyalgia?" and in French, "Souffrez-vous d'arthrite ou de rhumatisme, sauf la fibromyalgie?" In 2007 the question was simplified to, "Do you have arthritis, excluding fibromyalgia?" and in French, "Souffrez-vous d'arthrite, sauf la fibromyalgie?" For one year in 2011 only, there was a variation in the wording of the question in French: "Faites-vous de l'arthrite ou de l'arthrose, excepté la fibromyalgia?" In 2015, there was a major revision of the arthritis question. The revised question in English was, "Do you have arthritis, for example osteoarthritis, rheumatoid arthritis, gout or any other type, excluding fibromyalgia?" and in French, "Souffrez-vous d'arthrite, par exemple l'arthrose, l'arthrite rhumatoide [sic], la goutte ou toute autre forme d'arthrite, excluant la fibromyalgie?"

Sociodemographic variables. Age was categorized as 15-24, 25-34, 35-44, 45-54, 55-64, 65-74, 75-84, and 85+ years. "Low" education was defined as having secondary school education or less. Reported total household income from all sources in the past 12 months was coded as low (Can\$20,000), middle-low $(\$ 20,000-\$ 39,999)$, middle-high $(\$ 40,000-\$ 59,999)$, and high income $(\$ 60,000)$. Body mass index [BMI; weight $(\mathrm{kg}) /$ height $\left.\left(\mathrm{m}^{2}\right)\right]$ was calculated from self-reported weight and height, except for pregnant women and individuals $<0.914 \mathrm{~m}$ or $>2.108 \mathrm{~m}$ in height $(<5 \%$ of the sample). BMI was collapsed and divided into underweight/normal weight $\left(\leq 24.9 \mathrm{~kg} / \mathrm{m}^{2}\right)$, overweight $\left(25-29.9 \mathrm{~kg} / \mathrm{m}^{2}\right)$, and obese $\left(\geq 30 \mathrm{~kg} / \mathrm{m}^{2}\right)^{5}$. Physical activity was based on reported frequency and amount of time (in blocks of $10 \mathrm{~min}$ or more) spent participating in a range of physical activities including leisure time and transportation activities. Statistics Canada used these data to derive an index of total daily energy expenditure values ( $\mathrm{kcal} / \mathrm{kg} / \mathrm{day})$ in metabolic equivalents (MET; a value of metabolic energy cost). The index categorizes respondents as inactive, moderately active, or active, which was divided into inactive versus at least moderately active, where inactive means no reported eligible participation in activities ${ }^{3}$.

Health-related variables. As indicated in the introduction, longterm health conditions were ascertained by presenting respondents with a list to which they could answer yes or no. These data were used to derive a comorbidity count $(1,2$, or $3+)$. The conditions included were back problems, asthma, high blood pressure, migraine, chronic obstructive pulmonary disease, diabetes, heart disease, cancer, stroke, scoliosis, osteoporosis, fibromyalgia, high cholesterol, sleep apnea, and chronic fatigue. Respondents were also asked whether they had mood or anxiety disorders as longterm health conditions. "Pain and discomfort" was divided into the following: pain does not limit activity, or pain limits all, some, or a few activities. Respondents reporting that they had trouble going to sleep or staying asleep at least some of the time were categorized as having trouble sleeping. Questions on activities of daily living asked about difficulties or needing help for health reasons; responses were divided into no difficulty or at least some difficulty. The number of consultations with a family doctor or general practitioner in the previous 12 months was divided into $\leq 4$ or $>4$ consultations. Questions were also asked about consultations with specialists, and responses were none or $\geq 1$ consultation.

Analysis. National arthritis prevalence estimates were derived for 2000-2016, and national and provincial arthritis prevalence estimates, overall and by age and sex, were derived for 2015 and 2016. Descriptive analyses were used to document the effect of arthritis by sex and decade of age. For the projections we applied the age- and sex-specific prevalence estimates from the CCHS 2015 to the Statistics Canada population projections for 2020 to 2040 in 5-year intervals to estimate the number of people with arthritis, and to derive the expected prevalence of arthritis.

Analyses were carried out using the available datasets at the Statistics Canada Research Data Centre (Toronto). The data used for this study were made available to researchers through a formally reviewed research proposal to Statistics Canada (contract no. 12-SSH-UTO-3430), and in view of this our Institutional Review Board waived the requirement for ethics approval. All analyses included the data weights provided by Statistics Canada, which accounted for sample design and included adjustments for nonresponse and poststratification. To account for clustering in the sample, bootstrap techniques were used to derive variance estimates and calculate CI for the yearly prevalence estimates.

\section{RESULTS}

To show the effect of the changes in wording of the arthritis question, Figure 1 presents the estimates of the prevalence and numbers of people with arthritis (age $\geq 15 \mathrm{yrs}$ ) from sequentially available CCHS datasets from 2000 onward, including the most recent data from 2016. Between 2000 and 2014 there were some fluctuations in prevalence of arthritis, which were in part related to minor changes in the wording of the English and French versions of the arthritis question. Overall in these years, the prevalence varied between $15.4 \%$ and $17.6 \%$. There was a significant increase in prevalence to over $20 \%$ for the 2015 and 2016 surveys with the revised wording of the arthritis question, which included examples of what is meant by arthritis. Conservatively, this change represents an increase in the estimated number of Canadians with arthritis from around 4.8 million to 6 million.

To further examine how the change in wording in 2015 affected the prevalence estimates, a comparison of arthritis prevalence estimates by age and sex for 2015 and 2007/08 (the year of data included in the 2010 PHAC report) is shown in Figure 2 (with supporting data in Supplementary Table 1, available with the online version of this article). As can be seen, the change in wording resulted in a higher arthritis prevalence in all age and sex groups, except for men aged $85+$ years. The proportion of people with arthritis within each age group (the prevalence) increases steeply with age for both men and women. However, for policy and advocacy purposes, the absolute number of people in the population with arthritis is more relevant. The solid black line in Figure 3 shows the estimated number of people with arthritis in 2015 in each age group. Given the underlying age structure of the Canadian population, the age group with the most people with arthritis was $60-64$ years. Overall, $56 \%$ of people with

Personal non-commercial use only. The Journal of Rheumatology Copyright (C) 2019. All rights reserved. 


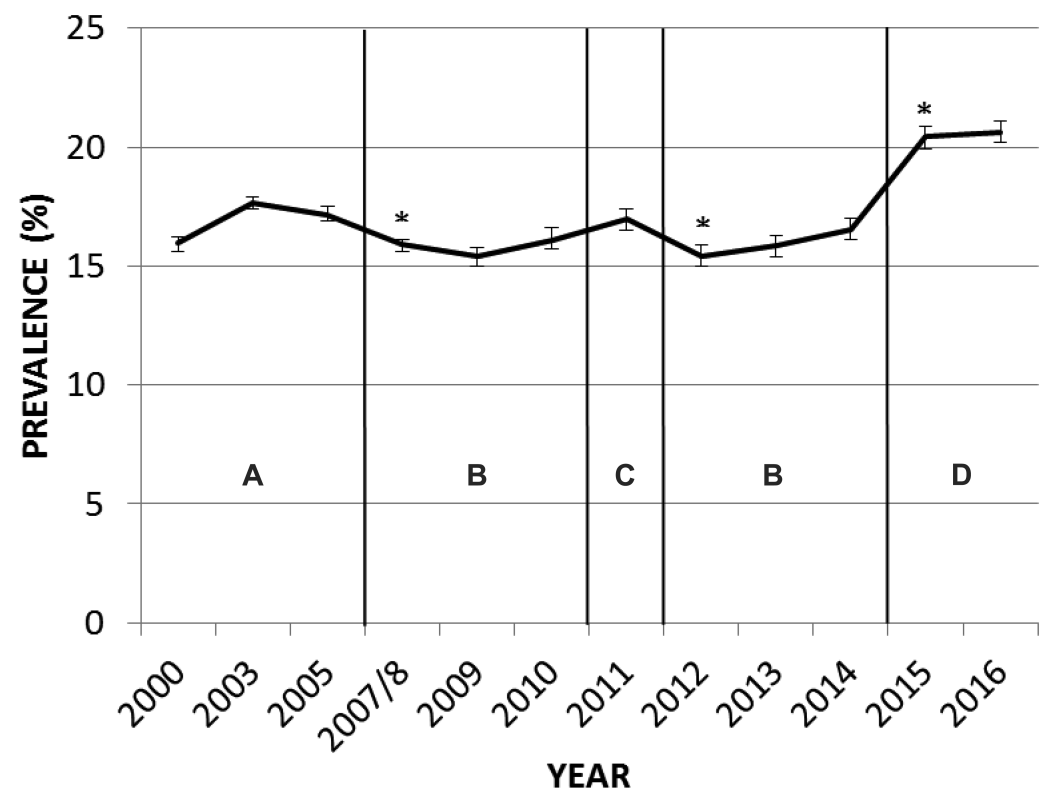

Figure 1. Estimated prevalence of arthritis in Canada (age $\geq 15$ yrs) 2000-2016. *Statistically significantly different from previous point (determined by non-overlapping bootstrapped 95\% CI). A. English: "Do you have arthritis or rheumatism, excluding fibromyalgia?" French: "Souffrez-vous d'arthrite ou de rhumatisme, sauf la fibromyalgie?" B. English: "Do you have arthritis, excluding fibromyalgia?" French: "Souffrez-vous d'arthrite, sauf la fibromyalgie?" C. English: Unchanged from B. French: "Faites-vous de l'arthrite ou de l'arthrose, excepté la fibromyalgia?" D. English: "Do you have arthritis, for example osteoarthritis, rheumatoid arthritis, gout or any other type, excluding fibromyalgia?" French: "Souffrez-vous d'arthrite, par exemple l'arthrose, l'arthrite rhumatoide, la goutte ou toute autre forme d'arthrite, excluant la fibromyalgie?"

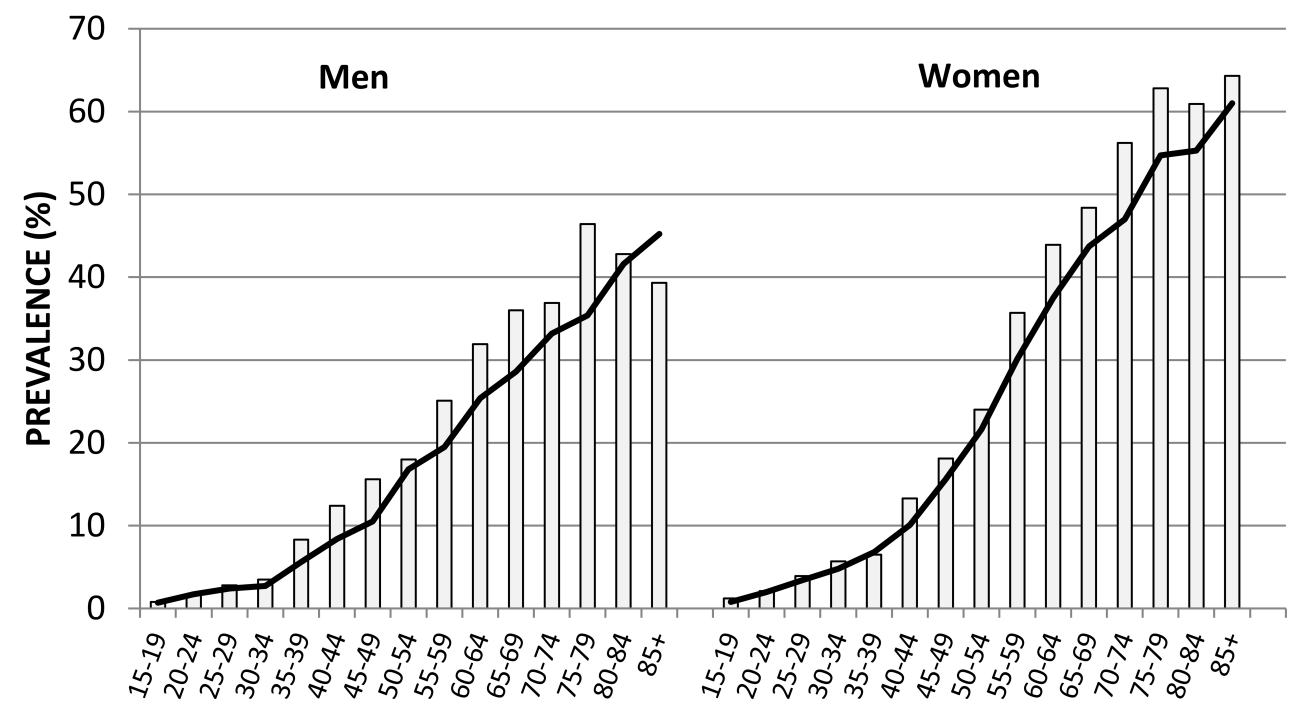

\section{AGE GROUP}

Figure 2. Prevalence of arthritis in Canada by age and sex comparing 2007/08 and 2015 data. CCHS: Canadian Community Health Survey; PHAC: Public Health Agency of Canada.

arthritis were aged $<65$ years, with 726,000 (12\%) aged between 15-44 years, and 2.6 million (44\%) aged 45-64 years. Table 1 gives national and provincial prevalence estimates and numbers with arthritis overall and by sex, and additionally provides estimates for the population aged 15-44, 45-64, and 65+ years for 2015 and 2016. Arthritis prevalence estimates were higher in the Eastern provinces, especially in Newfoundland and Nova Scotia. Quebec and

\section{Personal non-commercial use only. The Journal of Rheumatology Copyright (C) 2019. All rights reserved}




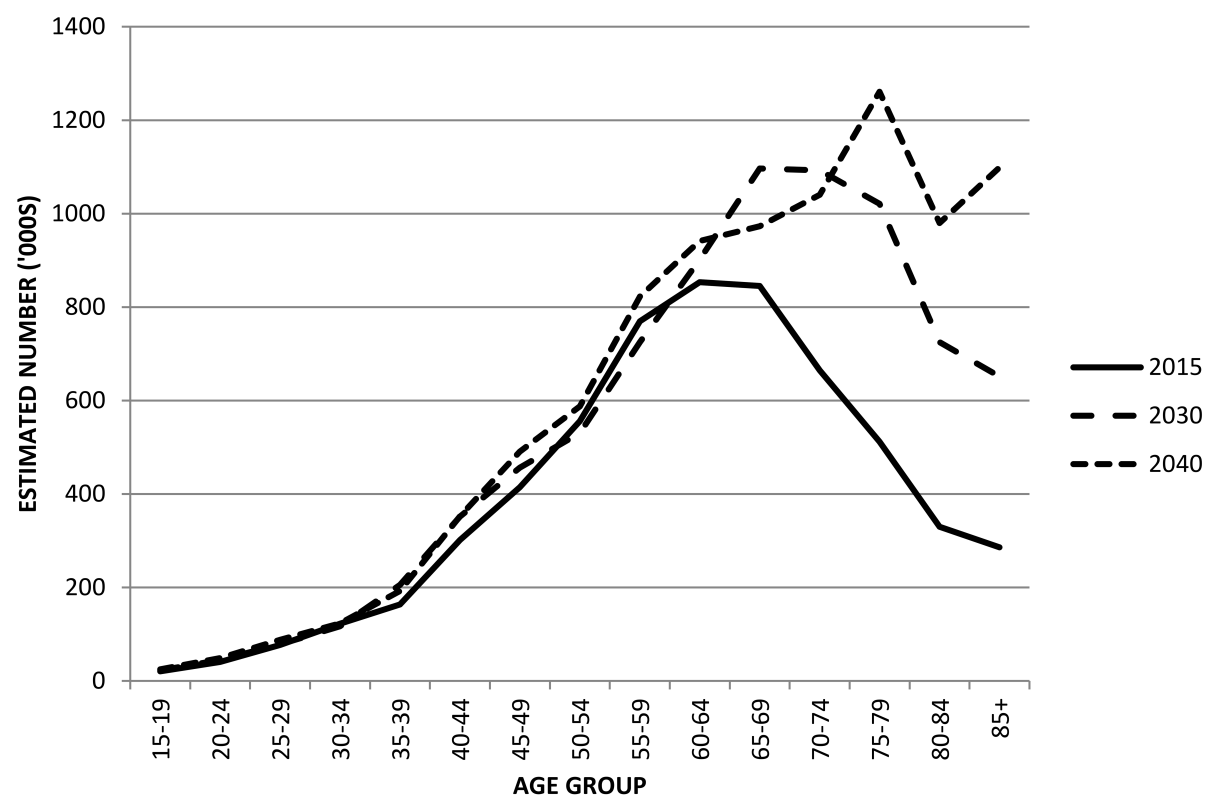

Figure 3. Estimated number of Canadians with arthritis by age group for 2015 and projections for 2030 and 2040.

Alberta had the lowest reported prevalence of arthritis in Canada. National estimates by 10 -year age groups and sex are provided in Supplementary Table 2 (available with the online version of this article).

An overall ranking of chronic conditions by prevalence showed that arthritis was the most frequent chronic condition in the Canadian population overall and among women; it was the third most frequent (after back problems and high blood pressure) among men (Figure 4). Table 2 shows selected key characteristics among individuals reporting a diagnosis of arthritis, by sex and by age. The upper part of the table summarizes the sociodemographic characteristics of people with arthritis. Most (65.8\%) were overweight or obese, and over a quarter were inactive with no appreciable physical activity. The lower part of the table summarizes multimorbidity and quality of life in people with arthritis, including visits to primary care physicians and specialists. Multimorbidity is a major issue: $82 \%$ of those with arthritis reported having at least 1 additional chronic condition, with one-third reporting 3+ conditions. Back problems, high blood pressure, high cholesterol, and diabetes were the most commonly reported. As expected, the extent of multimorbidity increased with age. Nevertheless, $3+$ additional chronic conditions were reported by $7 \%$ and $17 \%$ of individuals with arthritis, respectively, in the 15-24 and 25-34 age groups. Over 1 in 10 people with arthritis reported either mood or anxiety disorders, with almost one-quarter of those aged $<35$ years reporting these disorders. A high proportion of people with arthritis reported pain that prevented activities, trouble sleeping, and difficulties with activities of daily life. Over one-third made 4+ visits to primary care physicians, and two-fifths had at least 1 visit to a specialist. For most of these variables there was little variability by age.

National projections of the number of people who will have arthritis in 2030 and 2040 are presented in Figure 3 by age. By 2040, the overall prevalence is expected to increase to just over $24 \%$ of the population (age $\geq 15 \mathrm{yrs}$ ), with the number of Canadians living with arthritis projected to increase from 6 million in 2015 to 9 million in 2040. Supplementary Table 3 (available with the online version of this article) provides national projections of the number of people with arthritis and the expected prevalence of arthritis for 2020 to 2040 in 5-year intervals, overall and by sex.

\section{DISCUSSION}

The estimated national prevalence of arthritis was relatively stable between 2000 and 2014 but was substantially higher in 2015 and 2016, an increase that coincided with a change in the wording of the arthritis question. The revised question provided examples of 3 of the most frequent specific arthritis diagnoses, which likely reminded respondents of what was included under the arthritis banner. In addition, the French version of the question explicitly differentiated between rheumatoid (inflammatory) arthritis, arthrite, and osteoarthritis (OA), arthrose; with the exception of the 2011 survey, the earlier versions of the survey did not make this distinction, asking only about arthrite. This change may have contributed to the overall increase in prevalence, particularly in the province of Quebec, where the estimate increased from $11.8 \%$ in $2007 / 08$ to $18.5 \%$ in $2015^{1}$. Even with this increase, the prevalence of arthritis in Quebec was lower than in any other province, a finding that is consistent

Personal non-commercial use only. The Journal of Rheumatology Copyright (C) 2019. All rights reserved. 
Table 1. Canadian national and provincial estimates (2015 and 2016) for prevalence and number of people with arthritis (age $\geq 15$ yrs).

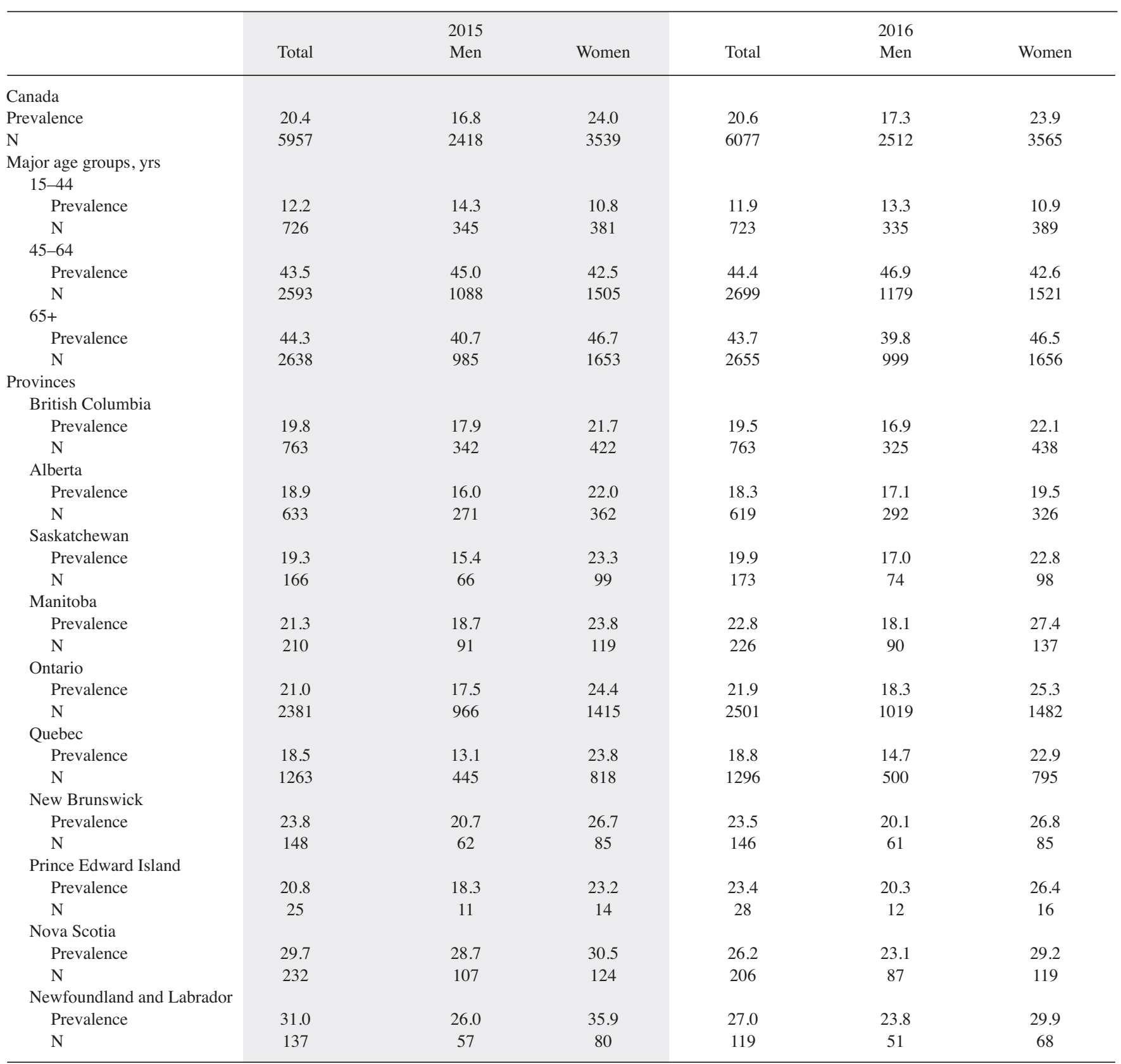

Data are expressed as prevalence (\%) and number (thousands).

with previous reports but for which contributing factors are unknown ${ }^{6}$. Overall these findings suggest that the prevalence of arthritis has previously been underestimated.

The sociodemographic and health-related characteristics of individuals reporting a diagnosis of arthritis from the 2015 CCHS are similar to those reported for 2007/08 in "Life with Arthritis in Canada"1 and aligns with other research, indicating that people with arthritis are at greater risk of having additional health conditions and poorer overall health ${ }^{1,7}$. The similarity of the effect in pain, trouble sleeping, and with activities of daily living across age and sex groups is consistent with previous findings ${ }^{8}$. This is contrary to what may be a common belief: that the effect of arthritis gets worse with age. Our projections of substantial increase in arthritis prevalence over time are compatible with those for the United States, which used a similar methodology for projections. The American estimate projected an almost $50 \%$ increase in the number of adults with arthritis between 2010 and $2040^{9}$. We project a just-over-50\% increase from 2015 to 2040. As illustrated in Figure 3, a driver of the increase is

Personal non-commercial use only. The Journal of Rheumatology Copyright @) 2019. All rights reserved. 


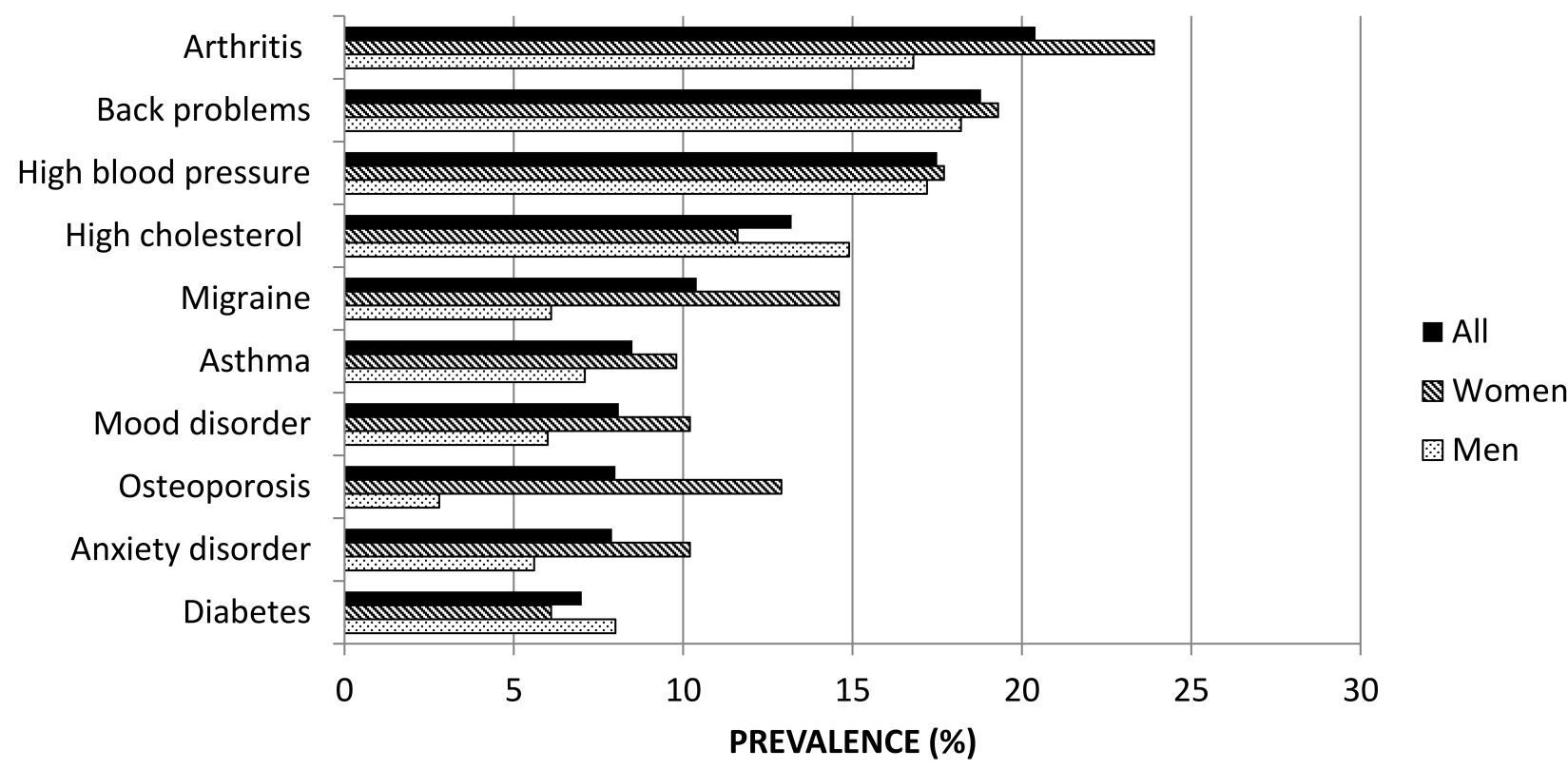

Figure 4. Ranking of top 10 chronic conditions by prevalence, Canada (2015).

the aging of the baby boomer population. The fact that more than half of "working age" individuals (aged 15-64 yrs) have arthritis is of particular concern given the potential economic effect of this longterm chronic condition on loss of productivity.

It is clearly not justifiable to dismiss arthritis as a condition of the elderly. The increase in arthritis prevalence with age means that the aging of the population will have a large influence on the number of older people with arthritis in coming decades. This likely will have implications for the provision of elder care, as well as economic consequences. For example, with the abolition of mandatory retirement, many people with arthritis may not be able to choose the opportunity to remain longer in the workforce owing to pain and disability. The strength of our study is that we have data on the questions used to ascertain arthritis from representative population health surveys from 2000 onward, so we can be fairly certain that the large increase in prevalence found from 2014 to 2015 is attributable to a change in the wording of the arthritis question. The major limitation is that all the data presented in this paper are self-reported. However, studies have shown that self-reports of arthritis diagnosis have an acceptable sensitivity and specificity for public health surveillance purposes ${ }^{10,11}$. Also, the questions concerning health-related characteristics, such as pain and sleep, and doctor visits were asked of all respondents and cannot be attributed directly to arthritis. Additionally, questions about difficulty in activities of daily living were asked only in the Maritime provinces.

A limitation with the prevalence projections is the underlying assumption that the age- and sex-specific prevalence of arthritis will remain stable into the future. However, examination of the age- and sex-specific prevalence from 2000 to 2014 showed that while there were some minor fluctuations over time, historically the estimates were essentially stable. Our projections relied on Statistics Canada projections for the growth of the Canadian population. These do not take into account possible changes in the composition of the population, for example, regarding obesity, ethnicity, and other risk factors for arthritis. Obesity is a major risk factor for knee $\mathrm{OA}$, one of the most frequent types of arthritis ${ }^{12,13}$. Arthritis also tends to be more prevalent in indigenous populations ${ }^{14}$ and African Americans ${ }^{15}$, and less prevalent in Asian populations ${ }^{14}$.

The 2015 estimated prevalence of arthritis in the Canadian population aged $\geq 15$ years is substantially higher than previous estimates, a change likely mainly due to changes in wording of the question asking about arthritis. With population growth and the aging baby boomer population, the prevalence is projected to continue increasing. Over the next decade, arthritis will place an even greater strain on the economy and on the healthcare system ${ }^{2}$, and funding allocations for arthritis healthcare and research will require careful and deliberate consideration as a result. The findings also reinforce the need for initiatives to reduce the pain and disability associated with arthritis ${ }^{1}$.

\section{ACKNOWLEDGMENT}

This project was carried out with the support of a service contract to the Arthritis Community Research and Evaluation Unit from The Arthritis Society of Canada. The authors thank the Statistics Canada Research Data Centres Program for providing access to the data file. We thank Jessica Wilfong for her help with finalizing the manuscript.

Personal non-commercial use only. The Journal of Rheumatology Copyright @ 2019. All rights reserved. 


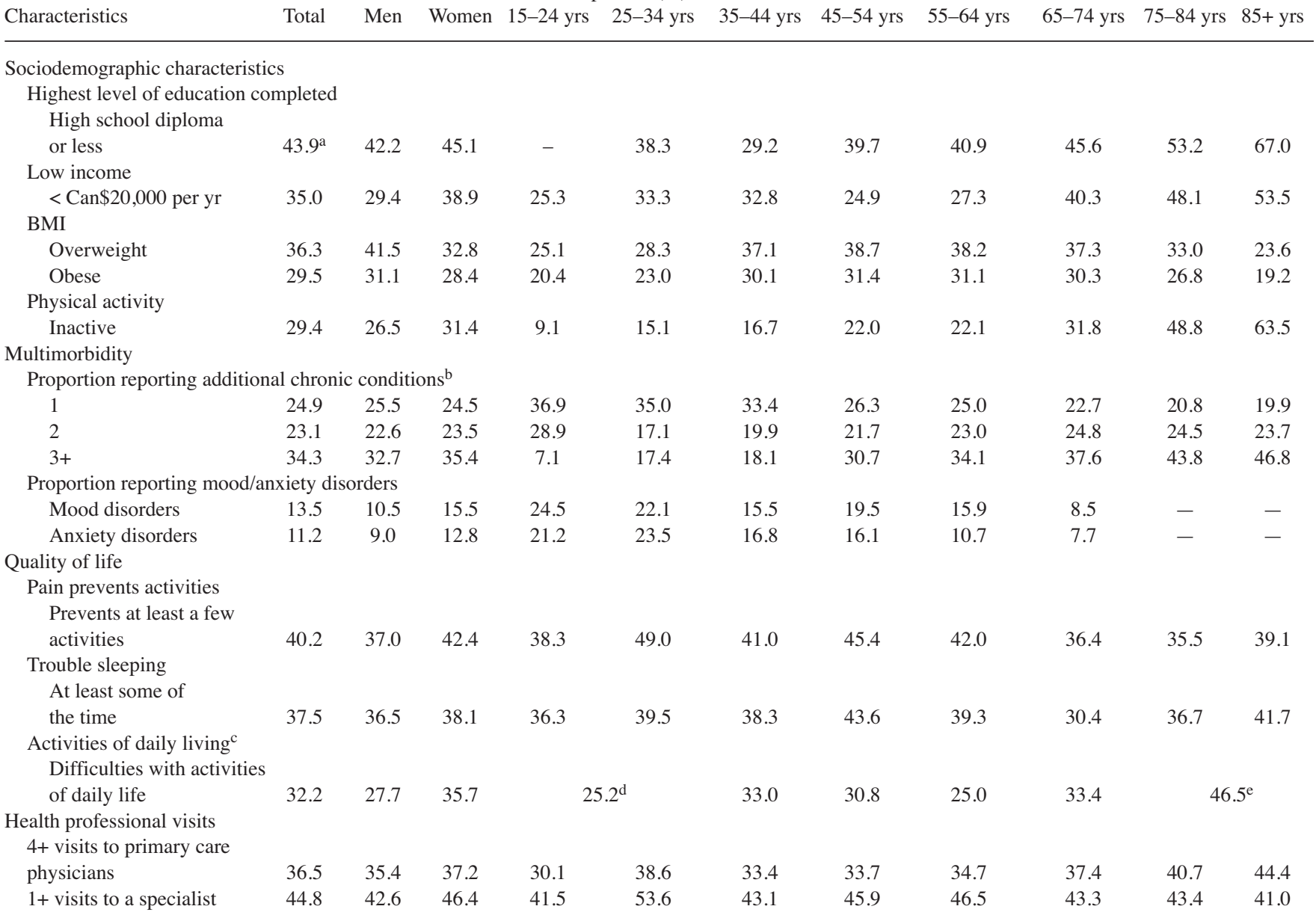

${ }^{a}$ Aged $20+$ yrs. ${ }^{b}$ List of chronic conditions: back problems, asthma, high blood pressure, migraine, chronic obstructive pulmonary disease, diabetes, heart disease, cancer, stroke, scoliosis, osteoporosis, fibromyalgia, high cholesterol, sleep apnea, and chronic fatigue. ${ }^{\mathrm{c}}$ Asked only in Newfoundland, Prince Edward Island, Nova Scotia, and New Brunswick. Questions asked about difficulty or needing help preparing meals, shopping for groceries or other necessities, doing everyday housework, doing heavy household chores (washing walls, yard work), personal care (washing, dressing), moving about the inside of the house, or paying bills. ${ }^{\mathrm{d}}$ Aged $15-34$ yrs. ${ }^{\text {e Aged }} 75+$ yrs. BMI: body mass index.

\section{ONLINE SUPPLEMENT}

Supplementary material accompanies the online version of this article.

\section{REFERENCES}

1. Public Health Agency of Canada. Life with arthritis in Canada: a personal and public health challenge. Ottawa: Public Health Agency of Canada; 2010.

2. Badley EM. Rheumatic diseases: the unnoticed elephant in the room. J Rheumatol 2008;35:6-7.

3. Statistics Canada. Canadian Community Health Survey - Annual Component (CCHS) - 2015. [Internet. Accessed December 13, 2018.] Available from: www23.statcan.gc.ca/imdb/p2SV.pl?Function=getSurvey\&Id= 238854

4. Statistics Canada. Canadian Community Health Survey - Annual Component (CCHS) - 2018. [Internet. Accessed December 13, 2018.] Available from: www23.statcan.gc.ca/imdb/p2SV.pl? Function=getSurvey \&amp;SDDS=3226
5. World Health Organization. Body mass index - BMI. [Internet. Accessed December 13, 2018.] Available from: www.euro.who.int/en/health-topics/disease-prevention/nutrition/ a-healthy-lifestyle/body-mass-index-bmi

6. Wang PP, Badley EM. Consistent low prevalence of arthritis in Quebec: Findings from a provincial variation study in Canada based on several Canadian population health surveys. J Rheumatol 2003;30:126-31.

7. Theis KA, Brady TJ, Helmick CG. No one dies of old age anymore: a coordinated approach to comorbidities and the rheumatic diseases. Arthritis Care Res 2017;69:1-4.

8. O'Donnell S, Rusu C, Hawker GA, Bernatsky S, McRae L, Canizares M, et al. Arthritis has an impact on the daily lives of Canadians young and old: Results from a population-based survey. BMC Musculoskelet Disord 2015;16:230.

9. Hootman JM, Helmick CG, Barbour KE, Theis KA, Boring MA. Updated projected prevalence of self-reported doctor-diagnosed arthritis and arthritis-attributable activity limitation among US adults, 2015-2040. Arthritis Rheumatol 2016;68:1582-7. 
10. Bombard JM, Powell KE, Martin LM, Helmick CG, Wilson WH. Validity and reliability of self-reported arthritis: Georgia senior centers, 2000-2001. Am J Prev Med 2005;28:251-8.

11. Sacks JJ, Harrold LR, Helmick CG, Gurwitz JH, Emani S, Yood RA. Validation of a surveillance case definition for arthritis. J Rheumatol 2005;32:340-7.

12. Silverwood V, Blagojevic-Bucknall M, Jinks C, Jordan JL, Protheroe J, Jordan KP. Current evidence on risk factors for knee osteoarthritis in older adults: a systematic review and meta-analysis. Osteoarthritis Cartilage 2015;23:507-15.

13. Reyes C, Leyland KM, Peat G, Cooper C, Arden NK,

Prieto-Alhambra D. Association between overweight and obesity and risk of clinically diagnosed knee, hip, and hand osteoarthritis: a population-based cohort study. Arthritis Rheumatol 2016; 68:1869-75.

14. Canizares M, Power JD, Perruccio AV, Badley EM. Association of regional racial/cultural context and socioeconomic status with arthritis in the population: A multilevel analysis. Arthritis Rheum 2008;59:399-407.

15. Jordan JM, Helmick CG, Renner JB, Luta G, Dragomir AD, Woodard J, et al. Prevalence of knee symptoms and radiographic and symptomatic knee osteoarthritis in African Americans and Caucasians: the Johnston County osteoarthritis project. J Rheumatol 2007;34:172-80. 